6 - ORIGINAL ARTICLE

MATERIALS TESTING

\title{
Reconstruction of abdominal wall defects using small intestinal submucosa coated with gelatin hydrogel incorporating basic fibroblast growth factor ${ }^{1}$
}

\author{
Liang Wang', Dong-ming Lai" ${ }^{\mathrm{II}}$, Bin Yang ${ }^{\mathrm{II}}$, Zhi-peng Jiang ${ }^{\mathrm{II}}$, Yu-chao Zhang ${ }^{\mathrm{II}}$, Jun Zhou ${ }^{\mathrm{II}}$, Wei Lai", Shuang Chen ${ }^{\mathrm{II}}$ \\ DOI: http://dx.doi.org/10.1590/S0102-86502014000400006
}

IPhD, Department of Surgical Disciplines, South China Training Center for Hernia Therapy, Sun Yat-sen Memorial Hospital, Sun Yat-sen University, Guangzhou, China. Design of the study, English language, manuscript writing, critical revision.

"IPhD, Department of Surgical Disciplines, South China Training Center for Hernia Therapy, Sun Yat-sen Memorial Hospital, Sun Yat-sen University, Guangzhou, China. Acquisition of data, critical revision.

IIIPhD, Full Professor, Department of Surgical Disciplines, South China Training Center for Hernia Therapy, Sun Yat-sen Memorial Hospital, Sun Yatsen University, Guangzhou, China. Design of the study, critical revision.

\begin{abstract}
PURPOSE: To construct a new biomaterial-small intestinal submucosa coated with gelatin hydrogel incorporating basic fibroblast growth factor, and to evaluate the new biomaterials for the reconstruction of abdominal wall defects.

METHODS: Thirty six Sprague-Dawley rats were used in the animal experiments and randomly divided into three groups. The new biomaterial was constructed by combining small intestinal submucosa with gelatin hydrogel for basic fibroblast growth factor release. Abdominal wall defects were created in rats, and repaired using the new biomaterials (group B), compared with small intestinal submucosa (group S) and ULTRAPRO ${ }^{\mathrm{TM}}$ mesh (group P). Six rats in each group were sacrificed at three and eight weeks postoperatively to examine the gross effects, inflammatory responses, collagen deposition and neovascularization.

RESULTS: After implantation, mild adhesion was caused in groups B and S. Group B promoted more neovascularization than group $\mathrm{S}$ at three weeks after implantation, and induced significantly more amount of collagen deposition and better collagen organization than groups $\mathrm{S}$ and $\mathrm{P}$ at eight weeks after implantation.
\end{abstract}

CONCLUSION: Small intestinal submucosa coated with gelatin hydrogel incorporating basic fibroblast growth factor could promote better regeneration and remodeling of host tissues for the reconstruction of abdominal wall defects.

Key words: Abdominal Wall. Biocompatible Materials. Intestinal, Fibroblast Growth Factor 2. Rats. 


\section{Introduction}

Reconstruction of the abdominal wall is important for extensive resections from surgery or large tissues that are lost during traumatic injuries ${ }^{1}$. Currently, there are many biological scaffold materials from human cadavers or other animal sources utilized for surgical treatment of abdominal wall defects, such as human acellular dermal matrix (HADM), small intestine submucosa (SIS) and so on. These biomaterials have been shown to facilitate the constructive remodeling of many different tissues in both preclinical animal studies and in human clinical applications, and they have been used in the field of abdominal wall reconstruction for many years. It has been reported that these biomaterials consist of a complex mixture of molecules that mediate structural and/ or biological properties, and also could effectively promoted cellular infiltration, new extracellular matrix deposition and neovascularization ${ }^{2,3}$. In addition, these biomaterials could become indistinguishable from native tissues. These characteristics might provide distinct advantages over synthetic meshes, especially in overcoming the postoperative complications, such as stiffness of abdominal wall, adhesions, chronic inflammation, foreign body reaction, and so on. However, the biological and mechanical properties of these biological scaffold materials are variable and complications still occur occasionally. Thus, the research for better biomaterials remains necessary.

It has been widely recognized that growth factors could greatly contribute to tissue regeneration at different stages of cell proliferation and differentiation ${ }^{4}$, and some members of the growth factor family have been tested in the prevention of wound failure and incisional hernia formation ${ }^{5,6}$. Among the growth factors essential for tissue regeneration is basic fibroblast growth factor (bFGF), which has been shown to be a potent stimulator of angiogenesis and cell migration and proliferation both in vivo and in vitro ${ }^{7}$. However, successful reconstruction of the abdominal wall induced by bFGF has not been always achieved. The most probable reason is the short half-life of bFGF in vivo. Therefore it is necessary to develop a drugdelivery system to gradually release bFGF in vivo and extend the biological activity. Recently, studies have shown that biodegradable gelatin hydrogels could incorporate bFGF and release it sustainedly in vivo to induce regeneration and reconstruction of host tissues ${ }^{8,9}$. This could be explained in terms of the basic-acidic interaction between the growth factor and gelatin hydrogels ${ }^{10}$. The acidic gelatin hydrogel could ionically immobilize in bFGF, and in vivo release of bFGF is mainly governed by hydrogel biodegradation ${ }^{11}$. That is, the growth factor release in vivo could be controlled by biodegradation of gelatin hydrogel itself.
In the present study, our aim was to combine small intestinal submucosa with a gelatin hydrogel incorporating basic fibroblast growth factor (bFGF-SIS) and to evaluate the effect of bFGF-SIS on the construction of abdominal wall defects in rats. We hypothesized that bFGF-SIS would enhance cellular and vascular infiltration and collagen formation from surrounding tissues and promote the regeneration and remodeling of host tissues for the reconstruction of abdominal wall defects after implantation.

\section{Methods}

All animal study protocols were reviewed and approved by the Institutional Animal Care and Use Committee of Sun Yat-Sen University (IACUC-2013-0101). Meanwhile, the experimental protocol was conformed to the guidelines of ethical use of animals, and suffering was made to minimize. Large swine (about 200kg) from a local slaughterhouse were used for the preparation of SIS. A total of 36 Sprague-Dawley rats (SD rats) weighing 240-280g, which were used for surgically created models of abdominal defects and histological and immunohistological analysis after the defect was repaired, were purchased from the Experimental Animal Center of Guangzhou Province, China. The rats were quarantined for one week prior to use and each rat was marked with an identification number. The rats were caged andW kept on a 12-hour light/12-hour dark schedule at $22^{\circ} \mathrm{C}$

A gelatin sample with an isoelectic point (IEP) of 4.75.2 , prepared from bovine bone through an alkaline process, was purchased from Sigma-Aldrich (St. Louis, MO, USA). Human recombinant bFGF with an IEP of 9.6 and a molecular weight of $16.0 \mathrm{kDa}$ was purchased from R\&D Systems, Inc. (Minneapolis, MN, USA). ULTRAPRO ${ }^{\mathrm{TM}}$ mesh, which is manufactured from approximately equal parts of absorbable poliglecaprone-25 monofilament fiber and non-absorbable polypropylene monofilament fiber, was obtained from Ethicon Inc. (Somerville, NJ, USA). Other chemicals were obtained from the Medical Research Center of Sun Yat-Sen Memorial Hospital, Sun Yat-sen University, Guangzhou, China.

\section{Preparation of gelatin hydrogels incorporating $b F G F$}

Gelatin hydrogels were prepared through the chemical cross-linking of gelatin solution with glutaraldehyde according to the method described previously ${ }^{12}$. In brief, the glutaraldehyde solution and solid gelatin were immersed into the mixed solution 
of gelatin $(5.0 \%)$ and glutaraldehyde $(0.1 \%)$, followed by leaving at $4^{\circ} \mathrm{C}$ for $15 \mathrm{~min}$ to allow them to crosslink and solidify. The resulting hydrogel sheets were punched out to obtain gelatin hydrogel discs of $10 \mathrm{~mm}$ in diameter and $2 \mathrm{~mm}$ in thickness, then left at $4^{\circ} \mathrm{C}$ for $12 \mathrm{~h}$ to allow the cross-linking reaction to proceed. After that, they were placed in $100 \mathrm{mM}$ glycine aqueous solution at $37^{\circ} \mathrm{C}$ for $1 \mathrm{~h}$ to block the residual aldehyde groups of glutaraldehyde, and then washed three times with double distilled water at $37^{\circ} \mathrm{C}$. To impregnate bFGF into the acidic gelatin hydrogels, $20 \mu \mathrm{l}$ of aqueous solution containing bFGF $(0.01 \mu \mathrm{g} / \mu \mathrm{l})$ was dropped onto a freeze-dried hydrogel, and then left at $4^{\circ} \mathrm{C}$ for $12 \mathrm{~h}$. Since the volume of growth factor solution was much smaller than that theoretically impregnated into the hydrogel, $100 \%$ of the growth factor added could be entirely incorporated into the hydrogel ${ }^{13}$.

\section{bFGF in vitro release test}

Release tests were conducted in $37^{\circ} \mathrm{C}$ incubator. The gelatin hydrogel disks impregnated with bFGF were put into a 6-well cell culture plate, and then $5 \mathrm{~mL}$ sterile phosphate buffered saline (PBS) was added to each well. After that, the wells were covered with a parafilm sheet to avoid medium evaporation. Medium was changed at predetermined time points ( 1 day, 3 days, 7 days, 10 days, 14 days, 21 days and 28 days) and the collected medium was placed in a $15 \mathrm{~mL}$ centrifuge tube and frozen at $-20^{\circ} \mathrm{C}$ prior to quantification in quadruplex experiments. The amounts of bFGF released were determined using an enzymelinked immuno-sorbent assay (EMD Biosciences, San Diego, USA). The optical density was determined at a wavelength of $450 \mathrm{~nm}$ with reference of $570 \mathrm{~nm}$.

\section{Preparation of SIS}

SIS was obtained from the proximal jejunum of large swine and manufactured as previously described ${ }^{14}$. Briefly, after the small intestine was harvested, the mucosal, muscular layers and serosa were mechanically removed, leaving the submucosa and basilar layers of the tunica mucosa, which has been identified as SIS. The submucosa was then cut into $10 \mathrm{~cm}-$ long sections that were processed through a series of chemical cleaning steps, by incubation in ethylenediaminetetraacetic acid (EDTA), $\mathrm{NaOH}, \mathrm{HCl}$, and $\mathrm{NaCl}$. Four layers of SIS were mechanically made by vacuum pressing to create a multilaminate device. The four layers SIS device was stored at $-80^{\circ} \mathrm{C}$ and thawed as needed. All SIS that were used for coating gelatin hydrogel incorporating bFGF and repairing the abdominal wall defects were four layers SIS.

\section{Construction of bFGF-SIS}

Firstly, the SIS was immersed into a mixed solution of gelatin $(5.0 \%)$ and glutaraldehyde $(0.1 \%)$, left at $4^{\circ} \mathrm{C}$ for $15 \mathrm{~min}$ (repeated three times), and then left at $4^{\circ} \mathrm{C}$ for $12 \mathrm{~h}$. Secondly, gelatin-coated SIS was placed in $100 \mathrm{mM}$ glycine aqueous solution at $37^{\circ} \mathrm{C}$ for $1 \mathrm{~h}$, and then washed three times with double-distilled water. Finally, the gelatin-coated SIS was freeze-dried and sterilized with ethylene oxide gas, stored at $-80^{\circ} \mathrm{C}$, and thawed as needed.

To impregnate bFGF with gelatin-coated SIS, $20 \mu 1$ of aqueous solution containing bFGF $(0.25 \mu \mathrm{g} / \mu \mathrm{l})$ was dropped onto the freeze-dried gelatin-coated SIS at room temperature, and then left at $4^{\circ} \mathrm{C}$ for $12 \mathrm{~h}$ to obtain the gelatin hydrogel incorporating bFGF.

\section{Surgical procedures and grouping}

A total of 36 rats were randomly divided into three groups. Pentobarbital sodium $(40 \mathrm{mg} / \mathrm{kg})$ was used for intraperitoneal anesthesia. After the rats were shaved, scrubbed, disinfected and covered with sterile draping, a $2.0 \times 1.5 \mathrm{~cm}$ full thickness defect in the anterior abdominal wall was surgically created, including subcutaneous tissue, fascia, muscle and the peritoneum. Then, the defects were primarily repaired with bFGF-SIS (group B), SIS (group S) or ULTRAPRO ${ }^{\mathrm{TM}}$ mesh (group P) respectively, and each implant oversized the defect by at least $0.25 \mathrm{~cm}$. The implant was used in the onlay position by fixing to the defect edge of abdominal wall with 4/0 monofilament polypropylene sutures (Prolene ${ }^{\circledR}$, Ethicon). Finally, the skin was closed with $2 / 0$ VWicryl sutures (Vicryl ${ }^{\circledR}$, Ethicon). Following recovery, each rat was returned to its cage and free access to food and drinking.

\section{Macroscopic examination of abdominal wall defect repairing}

All animals were checked three days after the implantation, and then weekly checked for local and systemic complications, including: death, wound infection, dehiscence, bulging or abdominal wall hernia and fistula formation. Six rats in each group were randomly sacrificed at three and eight weeks after the implantation, and the gross repair effects were observed and recorded before the biomaterials were sampled. 


\section{Adhesion evaluation}

For evaluation of the adhesion, six rats in each group were sacrificed at three and eight weeks postoperatively. A U-shaped incision was made in the abdominal wall, which included the biomaterial and its surrounding host tissue, without disturbing any adhesions. The degree of adhesions was assessed by two surgeons who were blinded to the study. The score of adhesions was obtained from each rat according to the criteria described previously ${ }^{15}: 0$, no adhesions; 1, thin and filmy adhesions easily separable by blunt dissection; 2, definite localized adhesions; 3 , definite multiple visceral adhesions; and 4 , dense adhesions extending abdominal wall.

\section{Histology and immunohistochemistry}

After the rats were sacrificed, the abdominal wall was removed en bloc for histology and immunohistochemistry examination. The specimens were fixed in 10\% formaldehyde immediately after explantation, embedded in paraffin, and cut into $5 \mu \mathrm{m}$ sections, and each slide would contain the biomaterials, interface, as well as the surrounding native tissue. For the histological examination, the slides were stained with hematoxylin and eosin (HE) and examined under a light microscope (E600, Nikon, Japan) to quantitatively analyze the number of foreign body giant cells (FBGCs), polymorphonuclear cells (PMNCs) and mononuclear cells (MNCs) invading the implanted scaffolds. Fields were randomly selected at the interface between the implant and the surrounding tissue. Semi-qualitative assessment of collagen deposition was performed using Masson's Trichrome stain. The amount of inflammatory cell infiltration and the amount and organization of collagen deposition were scored using a method similar to that described by Badylak et al. ${ }^{16}$. and Konstantinovic et al. ${ }^{17}$ (Table 1).

TABLE 1 - Histological scoring criteria used for microscopic examination.

\begin{tabular}{lcccc}
\hline \multirow{2}{*}{$\begin{array}{l}\text { Cellular } \\
\text { infiltration }\end{array}$} & \multicolumn{4}{c}{ Score } \\
\cline { 2 - 5 } & 0 & 1 & 2 & 3 \\
\hline FBGCs & 0 & $1-5$ & $6-10$ & $>10$ \\
PMNCs & 0 & $1-5$ & $6-10$ & $>10$ \\
MNCs & 0 & $1-5$ & $6-10$ & $>10$ \\
Collagen & & & & \\
Organiza- & Totally & Slightly & Moderately & Well \\
tion & disorganized & organized & organized & organized \\
Amount & None & Mild & Moderate & Abundant \\
Vascularity & 0 & $1-3$ & $4-10$ & $>10$ \\
\hline
\end{tabular}

FBGCs, Foreign body giant cells

PMNCs, Polymorphonuclear cells

MNCs, Mononuclear cells
Five non-overlapping fields per slide and two main slides were counted at $\times 400$ magnification in a standardized and blinded fashion.

The neovascularizition were assessed using immunohistochemical staining of CD31 (neointimal cells) (Mouse -anti rat monoclonal antibody, 1:200; Abcam, Cambridge, USA). Sections incubated without the primary antibody served as negative controls. The mean percentage area of blood vessels (\% Abv) was calculated for 10 randomly selected high-contrast fields (five non-overlapping fields per slide and two main slides) at $\times 200$ magnification using Image-Pro Plus (version 6.0, Media Cybernetics Inc, Silver Spring, MD, USA). Percentage area of blood vessels = area of capillary vessels / total tissue area $\times 100 \%$. All evaluations were performed by one pathologist who was blinded to the study design.

\section{Statistical analysis}

Experimental results were expressed as mean \pm standard deviation. Statistical analysis was carried out using SPSS version 16.0 for Windows (SPSS, Inc., Chicago, IL, USA). We performed statistical analyses using the one-way ANOVA, LSD-t test, KruskalWallis test, and the Mann-Whitney test. Values of $\mathrm{p}$ below 0.05 were considered to represent statistically significant difference.

\section{Results}

bFGF in vitro release

In the present study, the hydrogels were swollen gradually within the well and bFGF was released into the surrounding medium. Figure 1 shows the in vitro time profiles of bFGF release from gelatin hydrogel.

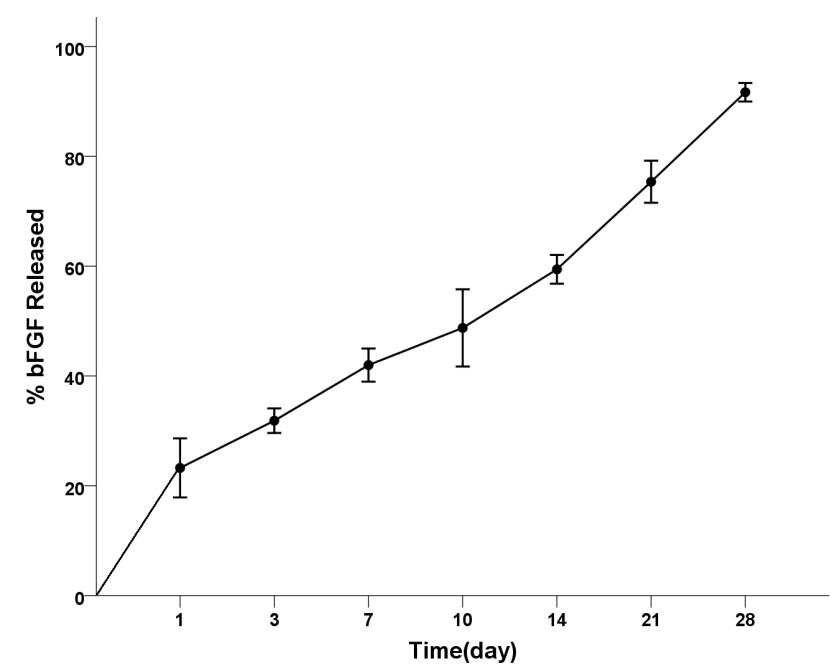

FIGURE 1 - Release profile of bFGF from gelatin hydrogels within 28 days. 
At the first day, the gelatin released $23.3 \% \pm 5.4 \%$ of bFGF, and the overall amount of bFGF released within 28 days was about $91.7 \% \pm 1.7 \%$ of its initial loading concentration.

\section{Macroscopic examination}

All rats survived to the predetermined sacrifice date. All the defects were repaired successfully, and none of the rats showed any evidence of bulging or herniation. None of rats developed wound rupture, wound infection or fistula formation postoperatively. Wound dehiscence was noted in one case in group $\mathrm{B}$, two cases in group $\mathrm{S}$ and two cases in group $\mathrm{P}$ at three days after implantation, all of which were disinfected and sutured then.

All the biomaterials used for the reconstruction of the abdominal wall defect remained visible three weeks after implantation (Figure 2A-C).

Eight weeks after implantation, bFGF-SIS and SIS were gradually integrated into the adjacent fascia tissues at the interface. Especially in group B, there was almost no distinction between bFGFSIS and the surrounding fascia tissues. The ULTRAPRO ${ }^{\mathrm{TM}}$ mesh remained visible compared with bFGF-SIS and SIS (Figure 2D-F).
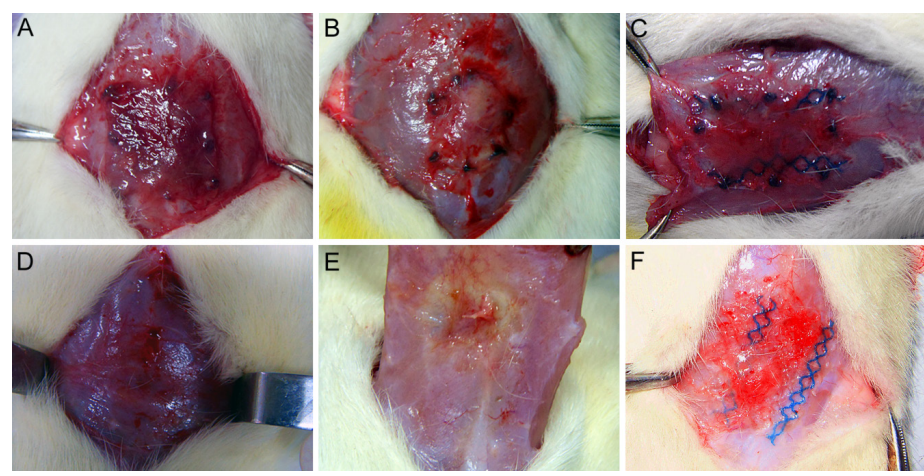

FIGURE 2 - Gross effects of three implants after implantation. (A) bFGFSIS, three weeks; (B) SIS, three weeks; (C) ULTRAPRO ${ }^{\text {TM }}$, three weeks; (D) bFGF-SIS, eight weeks; (E) SIS, eight weeks; (F) ULTRAPRO ${ }^{\mathrm{TM}}$, eight weeks.

\section{Adhesion analysis}

Three weeks after implantation, a lower degree of intraabdominal adhesion was observed in groups $\mathrm{B}$ and $\mathrm{S}$ compared to group $\mathrm{P}(\mathrm{p}<0.01)$, and there was no significant differences between groups B and S. Groups B and S showed thin and filmy adhesions, involving only omentum. However, adhesions seen in group $\mathrm{P}$ were firm and dense, which affected the omentum and bowel. Eight weeks after implantation, intra-abdominal adhesions in groups B and S were still slight and could be easily separated, whereas group $\mathrm{P}$ had more dense adhesions that needed a sharp dissection to separate. Differences in the adhesion scores between the three groups were significant (Figure 3).
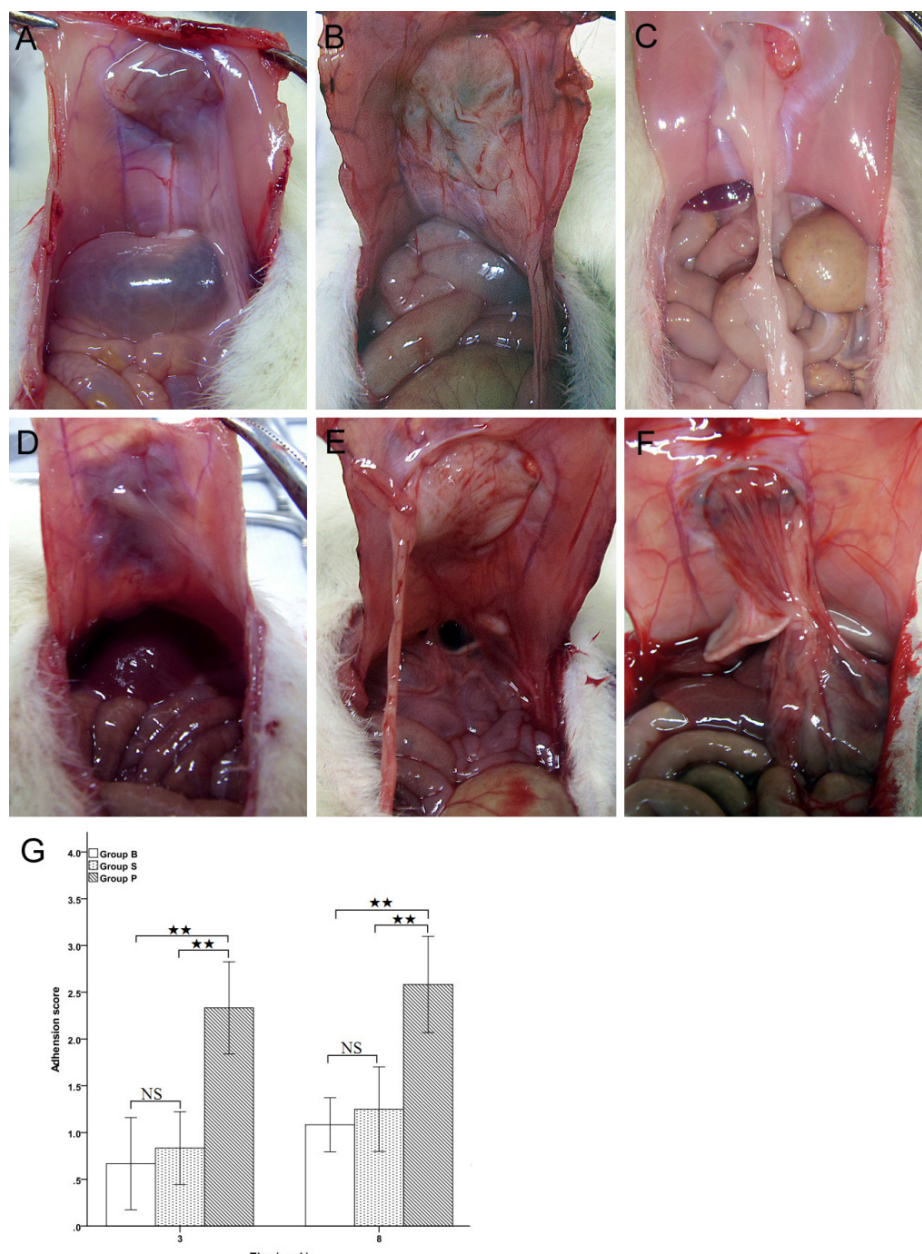

FIGURE 3 - Evaluation of intra-abdominal adhesion of three implants after implantation. (A) bFGF-SIS, three weeks; (B) SIS, three weeks; (C) ULTRAPRO ${ }^{\mathrm{TM}}$, three weeks; (D) bFGF-SIS, eight weeks; (E) SIS, eight weeks; (F) ULTRAPRO ${ }^{\mathrm{TM}}$, eight weeks; $(\mathrm{G})$ adhesion scores in bFGF-SIS, SIS, and ULTRAPRO ${ }^{\text {TM }}$ groups at three and eight weeks after implantation. ${ }^{*} \mathrm{p}<0.05, * * \mathrm{p}<0.01$.

\section{Microscopic evaluation}

Three weeks after implantation, histological examination with HE staining showed that inflammatory cell infiltrations were detected in all three groups. Group P showed more pronounced inflammatory cell infiltration at the interface between the biomaterials and native tissues, which was significantly greater than that seen in groups $B$ and $S(p<0.01)$. The remodeling of the biomaterials was observed in groups $\mathrm{B}$ and $\mathrm{S}$ with newborn blood vessels formation and new collagen deposition, which was less organized around the biomaterials. Furthermore, partial degradation of bFGF-SIS and SIS was detected at three weeks after implantation. Much more newborn blood vessels and totally 
disorganized collagen deposition were detected in group P (Figure $4 \mathrm{~A}-\mathrm{C}$ and Figure $5 \mathrm{~A}-\mathrm{C}$ ).
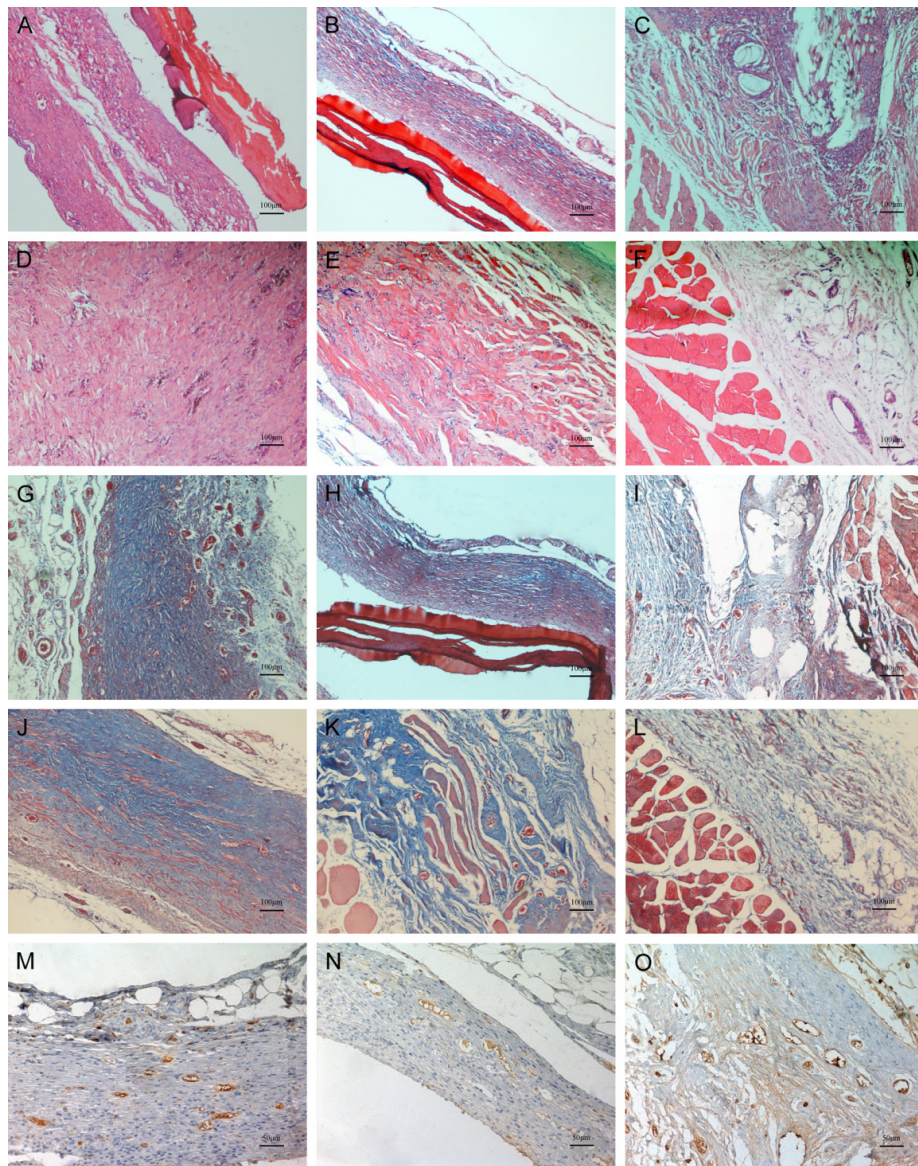

FIGURE 4 - Evaluation of histological appearance of three implants after implantation. (A-C) HE staining, three weeks, $\times 100$. A, bFGFSIS. B, SIS. C, ULTRAPRO ${ }^{\mathrm{TM}}$. (D-F) HE staining, eight weeks, $\times 100$. A, bFGF-SIS. B, SIS. C, ULTRAPRO ${ }^{\mathrm{TM}}$. (G-I) Masson's Trichrome staining, three weeks, $\times 100$. A, bFGF-SIS. B, SIS. C, ULTRAPRO ${ }^{\mathrm{TM}}$. (J-L) Masson's Trichrome staining, eight weeks, $\times 100$. A, bFGF-SIS. B, SIS. C, ULTRAPRO ${ }^{\mathrm{TM}}$. (M-O) Immunostaining of anti CD31 antibody. Three weeks, $\times 200$. A, bFGF-SIS. B, SIS. C, ULTRAPRO ${ }^{\mathrm{TM}}$
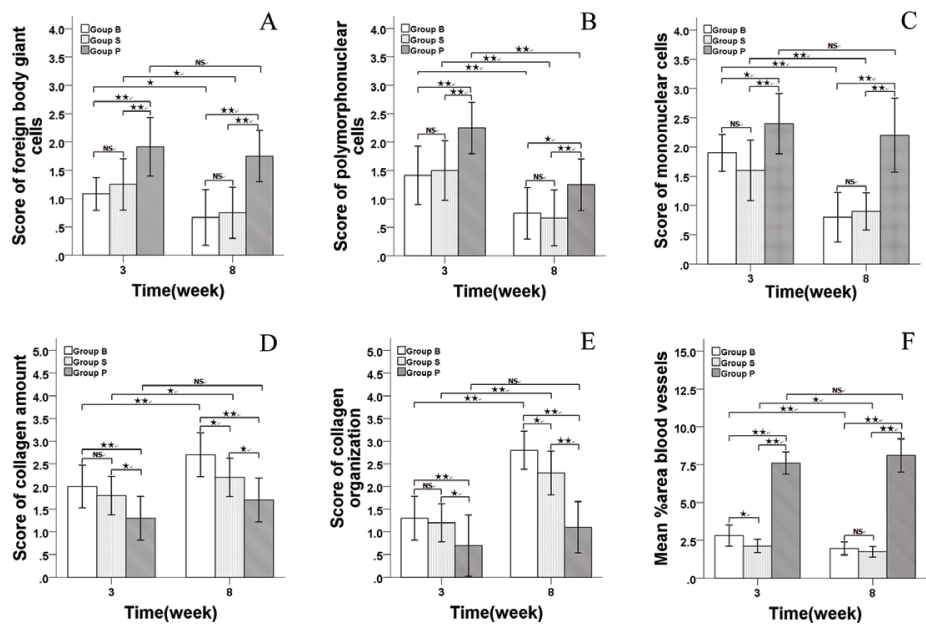

FIGURE 5 - Histological scores of three implants after implantation. (A) Statistical analysis of the scores for the number of FBGSs; (B) PMNs; (C) MNs; (D) amount of collagen; (E) organization of collagen; (F) amount of neovascularization. ${ }^{*} \mathrm{p}<0.05,{ }^{*} \mathrm{p}<0.01$.
At eight weeks after implantation, the inflammatory reactions gradually decreased in groups $\mathrm{B}$ and $\mathrm{S}$, along with more collagen deposition around the biomaterials, both of which were organized orderly and incorporated into the host tissues. However, few blood vessels were observed in both groups, compared with that seen at three weeks after the implantation. Group P showed no sign of mesh degradation, and inflammatory cell infiltration were still obvious. In addition, there were more newborn blood vessels with no sign of regression in Group $\mathrm{P}$, compared with groups $\mathrm{B}$ and $\mathrm{S}$ (Figure 4D-F and Figure 5A-C).

Masson's Trichrome staining showed that both groups B and $\mathrm{S}$ had a similar degree of collagen deposition and collagen organization over three weeks after implantation $(p>0.05)$. However, the scores of both amount and organization of collagen deposition in group B were higher than that in group $\mathrm{S}$ at eight weeks after the implantation $(\mathrm{p}<0.05)$. Both groups $\mathrm{B}$ and $\mathrm{S}$ showed more amount and better organization of collagen deposition than group $\mathrm{P}$ from three to eight weeks after implantation $(\mathrm{p}<0.01$, Figure 4G-L and Figure 5D-E).

Immunohistochemical staining with anti-CD31 showed that the density of newborn blood vessels in group B was significantly higher than that in group $\mathrm{S}$ at three weeks after implantation $(p<0.05)$. Gradually, with the maturation of regenerated collagen fibers, spontaneous regression occurred in the newborn blood vessel, and there were decreased newborn blood vessels in both groups $\mathrm{B}$ and $\mathrm{S}$ at eight weeks after implantation. There were much more newborn capillaries in Group $\mathrm{P}$ than in both groups $\mathrm{B}$ and $\mathrm{S}$ from three to eight weeks postoperatively (Figure 4M-O and Figure 5F).

\section{Discussion}

The increasing use of biomaterials for tissue defects repair has driven its development. Among the biomaterials used for the reconstruction of the abdominal wall, synthetic biomaterials, such as polypropylene, polytetrafluoroethylene and polyester, only serve as a scaffold providing mechanical support. However, they could cause potential complications, such as infections, adhesions, bowel obstruction, chronic pain, and local stiffness of the abdominal wall. Biological meshes, also called tissue-derived biomaterials, are derived from humans or animals tissues. The predominant advantages of these biomaterials are that they could induce cellular infiltration, new extracellular matrix deposition and neovascularization and carry a lower risk of complications. However, problems are still unavoidable. In order to increase the effect of abdominal wall reconstruction, modifications and 
innovations on biomaterials are needed. Nowadays, modification focusing on providing biomaterials with bioactive molecules (e.g. growth factors) is an important and effective approach, because these bioactive molecules could contribute to cell proliferation and differentiation during different periods of tissue regeneration ${ }^{18}$.

Several studies have shown that bFGF could promote fibroblast proliferation, stimulate angiogenesis in the dermis, and healing of fascial tissues ${ }^{5,18}$. However, effective use of bFGF is not always achieved due to its rapid diffusion into body fluids and short half-life ${ }^{19,20}$. Furthermore, high doses of bFGF could cause severe side effects ${ }^{21,22}$. Therefore the need for a sustained exposure of growth factor at the defects site for better effect has led to the employment of delivery vehicles that facilitate a therapeutic, localized dose, provide a stabilizing matrix for the growth factor, and minimize toxic side effects usually associated with systemic administration. It is reported that bFGF molecules was found to be ionically complexed with the acidic gelatin ${ }^{11}$, so that bFGF could be released by hydrogel degradation. In the present study, the release kinetics of bFGF was determined in temperature-controlled $\left(37^{\circ} \mathrm{C}\right)$ conditions. Within 28 days, more than $90 \%$ of the total amount of bFGF was released into the surrounding medium. Similar to previous study ${ }^{11}$, the gelatin hydrogel tended to release bFGF in a burst manner at beginning ${ }^{10}$, that is because the impregnation condition of bFGF (e.g. at $4^{\circ} \mathrm{C}$ for $12 \mathrm{~h}$ ) into the acidic gelatin hydrogel is not sufficient enough to achieve $100 \%$ ionic complexation ${ }^{23}$. Thereafter, no burst release was observed, and the gelatin hydrogel could sustainedly release bFGF in 28 days.

Intra-abdominal adhesion is an important evaluation criterion for the construction of the abdominal wall, which usually causes bowel obstruction, chronic pain and fistula formation after implantation. It has been reported that bleeding and inflammation are two major reasons for adhesion formation during the operation, because of trauma and foreign body reaction, the plasminogen activator, which directly increased the deposition of fibrin matrix was suppressed ${ }^{24,25}$. Therefore reduction of bleeding during the operation and subsequent inflammation could be a way to prevent adhesion formation. Our results showed that both bFGF-SIS and SIS caused minimal intra-abdominal adhesion compared with ULTRAPRO ${ }^{\text {TM }}$ mesh. That is probably because with the degradation bFGF-SIS and SIS, both the biomaterials resulted in minimal inflammation response and foreign body reaction. Decreased inflammation could prevent severe adhesion formation. In addition, it revealed that SIS coated with gelatin hydrogel would not slow down its degradation.

The host response to biomaterials can be characterized by inflammation and foreign body reaction, the formation of collagen tissue, as well as neovascularization. In our study, histological observation showed that the host responses of bFGF-SIS and SIS were quite different from those observed with ULTRAPRO $^{\mathrm{TM}}$ mesh. At three weeks after implantation, both bFGF-SIS and SIS caused obviously inflammatory response around the implants site. With time passed, inflammatory response decreased, and both bFGF-SIS and SIS degraded largely, both of which were replaced by a large amount of organized collagen tissue. Particularly for bFGF-SIS, there was an improved remodeling process with more amount and better organized collagen tissue deposition than SIS. Conversely, ULTRAPRO ${ }^{\mathrm{TM}}$ mesh caused a pronounced inflammatory response with obviously infiltration of PMNs, MNs or FBGCs at both three and eight weeks after implantation. The collagen tissues deposited around ULTRAPRO ${ }^{\mathrm{TM}}$ mesh were totally disorganized.

Angiogenesis - new blood vessel formation, accompanies fibroblast proliferation and allows nutrients and healing factors to enter the wound space ${ }^{26}$. It is also essential for the growth of granulation tissue and fibrous tissue, which could resist the distractive force of abdominal walls, and ischemic conditions around the biomaterial site often delays wound healing. In present research, quantitative immunohistochemistry showed that bFGF-SIS could promote more newborn blood vessels than SIS at early stage after implantation. More angiogenesis could make for more amount and better organization of collagen deposition. After that, with the maturation of regenerated collagen tissues, spontaneous regression occurred in newborn blood vessel, and there were few capillaries in group $\mathrm{B}$ and $\mathrm{S}$, which was similar to normal facial tissues. These results indicated that bFGFSIS could promote the regeneration of host facial tissues, because bFGF could facilitate the proliferation of dermal fibroblasts, vascular endothelial and smooth muscle cells ${ }^{27-29}$.

Optimal doses of growth factors, which were used in wound healing to obtain maximum effect, are not definite for human beings or animals. Various doses from 1 ng to $200 \mu \mathrm{g}$ have been tested in studies, however, not only the application ways and doses but also the experimental results are heterogenous ${ }^{30-33}$. In addition, it is not clear whether or not the effects of bFGF on wound healing are dose-dependent. In present study, $5 \mu \mathrm{g}$ bFGF was used, and results revealed that bFGF-SIS could promote better regeneration and remodeling of abdominal wall fascia tissues. It is surely possible that higher doses of bFGF could induce better results, however, it is necessary to test different doses in further studies.

One limitation of the present study which should be mentioned is that we only assessed the short-term effects of the bFGF-SIS for reconstruction of abdominal wall defects, and longterm studies are needed. 


\section{Conclusion}

Small intestinal submucosa coated with gelatin hydrogel incorporating basic fibroblast growth factor could promote better regeneration and remodeling of host tissues for the reconstruction of abdominal wall defects.

\section{References}

1. De Coppi P, Bellini S, Conconi MT, Sabatti M, Simonato E, Gamba PG, Nussdorfer GG, Parnigotto PP. Myoblast-acellular skeletal muscle matrix constructs guarantee a long-term repair of experimental full-thickness abdominal wall defects. Tissue Eng. 2006;12(7):1929-36.

2. Chen B, Lin H, Wang J, Zhao Y, Wang B, Zhao W, Sun W, Dai J. Homogeneous osteogenesis and bone regeneration by demineralized bone matrix loading with collagen-targeting bone morphogenetic protein-2. Biomaterials. 2007;28(6):1027-35.

3. Han Q, Sun W, Lin H, Zhao W, Gao Y, Zhao Y, Chen B, Xiao Z, Hu W, Li Y, Yang B, Dai J. Linear ordered collagen scaffolds loaded with collagen-binding brain-derived neurotrophic factor improve the recovery of spinal cord injury in rats. Tissue Eng Part A. 2009;15(10):2927-35.

4. Stocum DL. New tissues from old. Science. 1997;276(5309):15.

5. Dubay DA, Wang X, Kuhn MA, Robson MC, Franz MG. The prevention of incisional hernia formation using a delayedrelease polymer of basic fibroblast growth factor. Ann Surg. 2004;240(1):179-86.

6. Franz MG, Kuhn MA, Nguyen K, Wang X, Ko F, Wright TE, Robson MC. Transforming growth factor beta(2) lowers the incidence of incisional hernias. J Surg Res. 2001;97(2):109-16.

7. Folkman J, Klagsbrun M. Angiogenic factors. Science. 1987;235(4787):442-7.

8. Taipale J, Keski-Oja J. Growth factors in the extracellular matrix. FASEB J. 1997;11(1):51-9.

9. Tabata Y, Yamada K, Miyamoto S, Nagata I, Kikuchi H, Aoyama I, Tamura M, Ikada Y. Bone regeneration by basic fibroblast growth factor complexed with biodegradable hydrogels. Biomaterials. 1998;19(7-9):807-15.

10. Yamamoto M, Ikada Y, Tabata Y. Controlled release of growth factors based on biodegradation of gelatin hydrogel. J Biomater Sci Polym Ed. 2001;12(1):77-88.

11. Muniruzzaman, Tabata Y, Ikada Y. Complexation of basic fibroblast growth factor with gelatin. J Biomater Sci Polym Ed. 1998;9(5):459-73.

12. Yamamoto M, Tabata Y, Hong L, Miyamoto S, Hashimoto N, Ikada $\mathrm{Y}$. Bone regeneration by transforming growth factor $\beta 1$ released from a biodegradable hydrogel. J Control Release. 2000;64(13): $133-42$.

14. Takaoka R, Hikasa Y, Tabata Y. Vascularization around poly(tetrafluoroethylene) mesh with coating of gelatin hydrogel incorporating basic fibroblast growth factor. J Biomater Sci Polym Ed. 2009;20(10):1483-94.

15. Abraham GA, Murray J, Billiar K, Sullivan SJ. Evaluation of the porcine intestinal collagen layer as a biomaterial. J Biomed Mater Res. 2000;51(3):442-52.

16. Walker AP, Henderson J, Condon RE. Double-layer prostheses for repair of abdominal wall defects in a rabbit model. J Surg Res. 1993;55(1):32-7.

17. Badylak S, Kokini K, Tullius B, Simmons-Byrd A, Morff R. Morphologic study of small intestinal submucosa as a body wall repair device. J Surg Res. 2002;103(2):190-202.
18. Konstantinovic ML, Lagae P, Zheng F, Verbeken EK, De Ridder D, Deprest JA. Comparison of host response to polypropylene and non-cross-linked porcine small intestine serosal-derived collagen implants in a rat model. BJOG. 2005;112(11):1554-60.

19. Montesano R, Vassalli JD, Baird A, Guillemin R, Orci L. Basic fibroblast growth factor induces angiogenesis in vitro. Proc Natl Acad Sci USA. 1986;83(19):7297-301.

20. Zhao W, Chen B, Li X, Lin H, Sun W, Zhao Y, Wang B, Zhao Y, Han Q, Dai J. Vascularization and cellularization of collagen scaffolds incorporated with two different collagen-targeting human basic fibroblast growth factors. J Biomed Mater Res A. 2007;82(3):630-6.

21. Rapraeger AC, Krufka A, Olwin BB. Requirement of heparan sulfate for bFGF-mediated fibroblast growth and myoblast differentiation. Science. 1991;252(5013):1705-8.

22. Unger EF, Goncalves L, Epstein SE, Chew EY, Trapnell CB, Cannon RO 3rd, Quyyumi AA. Effects of a single intracoronary injection of basic fibroblast growth factor in stable angina pectoris. Am J Cardiol. 2000;85(12):1414-9.

23. Epstein SE, Fuchs S, Zhou YF, Baffour R, Kornowski R. Therapeutic interventions for enhancing collateral development by administration of growth factors: basic principles, early results and potential hazards. Cardiovasc Res. 2001;49(3):532-42.

24. Ikada Y, Tabata Y. Protein release from gelatin matrices. Adv Drug Deliv Rev. 1998;1(3):287-301.

25. Wasserberg N, Nunoo-Mensah JW, Ruiz P, Tzakis AG. The effect of immunosuppression on peritoneal adhesions formation after small bowel transplantation in rats. J Surg Res. 2007;141(2):294-8.

26. van't Riet M, de Vos van Steenwijk PJ, Bonthuis F, Marquet RL, Steyerberg EW, Jeekel J, Bonjer HJ. Prevention of adhesion to prosthetic mesh: comparison of different barriers using an incisional hernia model. Ann Surg. 2003;237(1):123-8.

27. Stadelmann W, Digenis A, Tobin G. Physiology and healing dynamics of chronic cutaneous wounds. Am J Surg. 1998;176(2A Suppl):26S-38S.

28. Arnold F, West D. Angiogenesis in wound healing. Pharmacol Ther. 1991;52 (3):407-22.

29. Krishnamoorthy L, Morris HL, Harding KG. Specific growth factors and the healing of chronic wounds. J Wound Care. 2001;10 (5):173-8.

30. Xie JL, Bian HN, Qi SH, Chen HD, Li HD, Xu YB, Li TZ, Liu XS, Liang HZ, Xin BR, Huan Y. Basic fibroblast growth factor (bFGF) alleviates the scar of the rabbit ear model in wound healing. Wound Repair Regen. 2008;16 (4):576-81.

31. Okazaki Y, Kagami H, Hattori T, Hishida S, Shigetomi T, Ueda M. Acceleration of rat salivary gland tissue repair by basic fibroblast growth factor. Arch Oral Biol. 2000;45(10):911-9.

32. Nurata H, Cemil B, Kurt G, Uçankuş NL, Dogulu F,Omeroğlu S. The role of fibroblast growth factor-2 in healing the dura mater after inducing cerebrospinal fluid leakage in rats. J Clin Neurosci. 2009;16 (4):542-4.

33. Iwakura A, Tabata Y, Nishimura K, Nakamura T, Shimizu Y, Fujita M, Komeda M. Basic fibroblast growth factor may improve devascularized sternal healing. Ann Thorac Surg. 2000;70(3):824-8.

34. Rieck P, Assouline M, Hartmann C, Pouliquen Y, Courtois Y. Effect of recombinant human basic fibroblast growth factor (rh-bFGF) on wound healing of the corneal epithelium. Ophthalmologe. 1993;90 (6):646-51.

\section{Acknowledgement}

To Yuru Fu, teacher of the Medical Research Center in Sun Yat-sen Memorial Hospital, Sun Yat-sen University for his assistance with the study. 
Wang L et al.

\section{Correspondence:}

\section{Shuang Chen}

Department of Surgical Disciplines

South China Training Center for Hernia Therapy

Sun Yat-sen Memorial Hospital

Sun Yat-sen University, Guangzhou 510120, China

Phone: +86-13808870507

Fax: +86-20-81332532

chensh2@mail.sysu.edu.cn

Received: Dez 10, 2013

Review: Feb 12, 2014

Accepted: March 14, 2014

Conflict of interest: none

Financial source: none

${ }^{1}$ Research performed at Laboratory Animal Center and Medical Research

Center, Sun Yat-sen Memorial Hospital, Sun Yat-sen University, China. 